\title{
Master-Slave Control for a Tele-Operation System of Construction Robot
}

\author{
(Improved Method of Control Compared with a Variable-Gain Symmetric-Position)
}

\author{
Hidetoshi KATO ${ }^{*}$, Hironao YAMADA ${ }^{* *}$ and Takayoshi MUTO ${ }^{* *}$ \\ *Graduate School of Engineering \\ Gifu University \\ ** Department of Mechanical and Systems Engineering \\ Gifu University, \\ 1-1 Yanagido, Gifu-shi, Gifu, 501-1193 Japan \\ (yamada@cc.gifu-u.ac.jp)
}

\begin{abstract}
This study deals with a master-slave system of a tele-operated construction robot. The system consists of an excavator as the construction robot, and two joysticks for operating the robot from a remote place. A fork glove is equipped with the front end of the excavator as a hand for grasping task objects. The master and the slave in this system correspond to, respectively, the fork glove and the joysticks controlled by an operator. In such a remote control system, the operator needs to feel a realistic sense of task force which is brought about from a feedback force of the fork glove. In order to attain an effective feedback of the task force, in the previous report of this study, a control method called a variable-gain symmetric-position was proposed. After that, however, a weak point was found in this method. Namely, the operator was not able to feel a realistic sense of the task force when he was grasping a soft object in comparatively a slow velocity. In the present report, therefore, for overcoming this problem, an improved method of control in comparison with the previous method is proposed.
\end{abstract}

\section{KEY WORDS}

Construction Machinery, Robot, Hydraulic Actuator, Master-slave control, Force feedback

\section{Introduction}

This study deals with a master-slave ${ }^{(1)}$ system of a tele-operated construction robot. The system consists of an excavator as the construction robot, and two joysticks for operating the robot from a remote place. A fork glove is equipped with the front end of the excavator as a hand for grasping task objects. The master and the slave in this system correspond to, respectively, the fork glove and the joysticks controlled by an operator. In such a remote control system, the operator needs to feel a realistic sense of task force which is brought about from a feedback force of the fork glove. In order to attain an effective feedback of the task force, in the previous report $^{(2)}$ of this study, a control method called a variable-gain symmetric-position was proposed. After that, however, a weak point was found in this method. Namely, the operator was not able to feel a realistic sense of the task force when he was grasping a soft object in comparatively a slow velocity. In order to overcome this problem, in this study, we propose an improved method to the previous one so that the operator can feel enough reaction force. By applying the new method to the master slave system, the usefulness of this method is confirmed 
in simulations and experiments.

\section{Nomenclature}

$a_{p}:$ cross-sectional area of piston

$\left[\mathrm{m}^{2}\right]$

$b$ : Viscous damping coefficient of piston $[\mathrm{Ns} / \mathrm{m}]$

$f_{s}$ : Driving force to slave

$k_{a}$ : Gain of servo amplifier

$K_{m}$ : Steady-state value of master

$k_{p m}, k_{d m}$ : Proportional and differential gains of master

$[\mathrm{Nm}],\left[\mathrm{Nm} / \mathrm{s}^{-1}\right]$

$k_{p s}, k_{d s}$ : Proportional and differential gains of slave

$k_{t m}:$ Torque gain of master $[\mathrm{m}],\left[\mathrm{m} / \mathrm{s}^{-1}\right]$

$[\mathrm{Nm}]$

$k_{x}, k_{p}$ : Flow rate gain, pressure gain $\left[\mathrm{m}^{2} / \mathrm{s}\right],\left[\mathrm{m}^{4} / \mathrm{kg}\right]$

$k_{v}$ : Gain of servo valve

$L_{m}:$ Dead time of master

$m$ : Mass of piston

$[\mathrm{kg}]$

$p_{a}, p_{b}$ : Pressures in piston chambers

$q_{L}, p_{L}$ : Flow rate, load pressure

$T$ : Gain

$T_{m}$ : Time constant of master

$u_{s}$ : Control input to slave

[V]

$x$ : Displacement of spool

[m]

[m]

$y_{m}, y_{s}$ : Displacements of master and slave

$y_{0 m}:$ Nominal quantity of $y_{m}\left(y_{0 m}=0.06\right)$

[m]

$Y_{m}$ : Nondimensional quantity of $y_{m}\left(=y_{m} / y_{0 m}\right)$

$y_{o s}$ : Nominal quantity of $y_{s}\left(y_{0 s}=0.3\right)$

$[-]$

$Y_{s}:$ Nondimensional quantity of $y_{s}\left(=y_{s} / y_{0 s}\right)$

$\zeta$ : Damping ratio

$[-]$
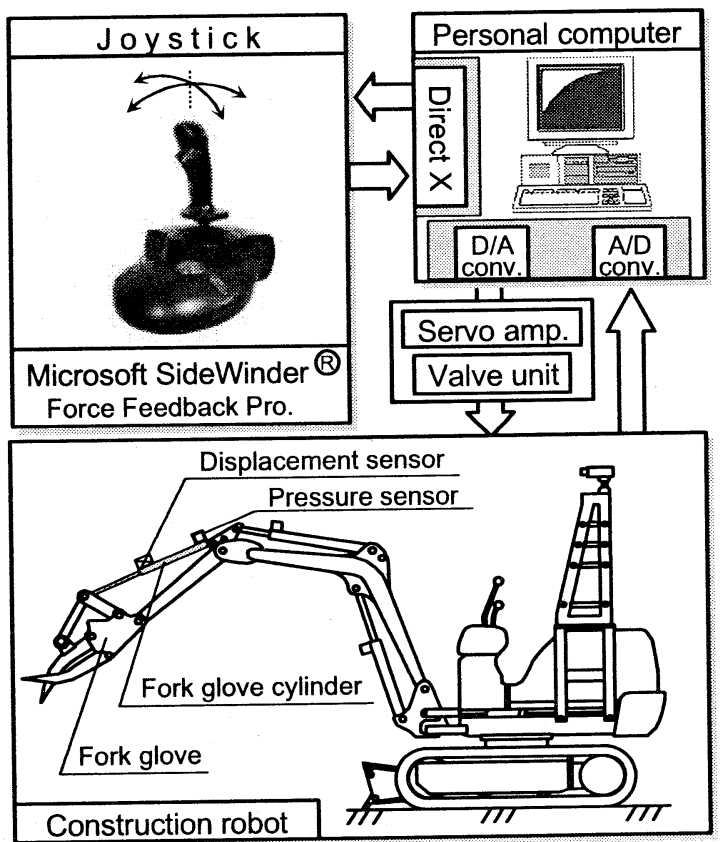

Figure 1 Schematic diagram of experimental apparatus $\tau_{m}:$ Input torque to joystick

[Nm]

$\tau_{r}:$ Reaction torque to joystick

[Nm]

$\tau_{0 r}$ : Nominal quantity of $\tau_{r}\left(\tau_{0 r}=0.82\right) \quad[\mathrm{Nm}]$

$T_{r}$ : Nondimensional quantity of $\tau_{r}\left(=\tau_{r} / \tau_{0 r}\right) \quad[-]$

$\omega_{n}:$ Natural angular frequency

$[\mathrm{rad} / \mathrm{s}]$

\section{Tele-operated construction robot system}

Figure 1 shows a schematic diagram of experimental apparatus consists of the joystick and the tele-operated construction robot. The joystick ("SideWinder Force Feedback 2", Microsoft Co.,Ltd.) can be operated to the $\mathrm{X}$ - and Y-axis directions. Displacements of the joystick are detected by position sensors. A DC motor is connected under the joystick, and the reaction force from the fork glove is fed back to the operator through a speed change gear connected to the DC motor. The hydraulic cylinders for driving the construction robot are controlled by the servo valves. The displacements of the cylinders are detected by magnetic stroke sensors which are embedded in the pistons. The external forces to which the cylinders respond are detected by a pair of pressure sensors attached to the cylinders.

4. Mathematical model for simulation analysis

In this chapter, the mathematical model of the master slave system is derived for the preparation of simulation analysis discussed later.

\subsection{Master system}

In order to get the mathematical model of the master system, we practiced a parameter identification of the joystick. The result obtained is shown in Fig. 2. The figure shows a step response curve of the displacement $y_{m}$ to the input torque of the joystick. From this result, the transfer function of the joystick was estimated as the one of the first order lag system $\left(T_{m}=0.125 \mathrm{~s}, K_{m}=0.18\right.$ $\left.\mathrm{N}^{-1}\right)$ with a time lag element $\left(L_{m}=0.08 \mathrm{~s}\right)$.

\subsection{Slave system}

In this study the same slave system is employed as in the previous paper. Therefore, the mathematical model ${ }^{(3)}$

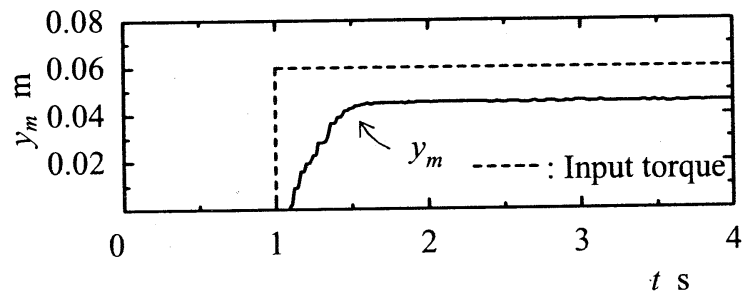

Figure 2 Step response of master system 
used for this slave system is also the same as in the previous one. The following assumptions are made.

(1) The compressibility of oil is neglected.

(2) Unsteady flows in the pipelines which connect the servo valve and the cylinder are neglected.

(3) The servo valve is a zero-lap type (ideal valve), and the flow coefficient is constant.

(4) Two cross-sectional areas of the single rod piston are expressed by the averaged value $a_{p}$.

(5) The coulomb friction is neglected.

The equation of motion of the servo valve is given by Eq. (1).

$$
\ddot{x}+2 \zeta \omega_{n} \dot{x}+\omega_{n}^{2} x=k_{a} k_{v} \omega_{n}^{2} u_{s}
$$

The equation of motion of the piston is given by Eq. (2).

$$
\left.\begin{array}{c}
m \ddot{y}_{s}+b \dot{y}_{s}=a_{p} p_{L} \\
p_{L}=p_{a}-p_{b}
\end{array}\right\}
$$

The relations for flow rate $q_{L}$ are given by Eqs. (3) and (4).

$$
\begin{aligned}
& q_{L}=k_{x} x-k_{p} p_{L} \\
& q_{L}=a_{p} \dot{y}_{s}
\end{aligned}
$$

Consequently the mathematical model of the slave system is represented by Eq. (1) to Eq. (4).

\section{Master-slave system}

5.1 Control method of variable-gain symmetric-position. In this part, we summarize the previous method ${ }^{(2)}$ of variable-gain symmetric-position. The block diagram for representing the control method is shown in Fig. 3. In this method, the reaction torque to the joystick $\tau_{r}$ is given by Eq. (5).

$$
\begin{gathered}
\tau_{r}=T k_{p m}\left(Y_{s}-Y_{m}\right)+T k_{d m}\left(\dot{Y}_{s}-\dot{Y}_{m}\right) \\
T=\left\{\begin{array}{cc}
0 & \left(f_{e} \leq f \leq f_{c}\right) \\
0<\frac{f-f_{e}}{f_{e_{-} \max }-f_{e}}<1 & \left(f<f_{e}\right) \\
0<\frac{f-f_{c}}{f_{c_{-} \max }-f_{c}}<1 & \left(f>f_{c}\right)
\end{array}\right.
\end{gathered}
$$

and $f_{e} / f_{c}$ : driving forces to the cylinder in expanding/contracting motions when an external force generated for grasping a task object by the fork glove is

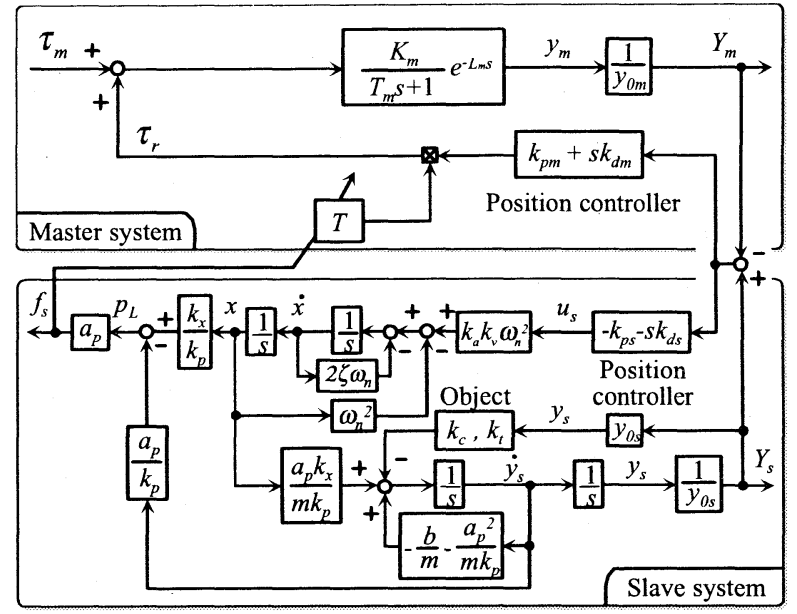

Figure 3 Block diagram of variable-gain symmetricposition control

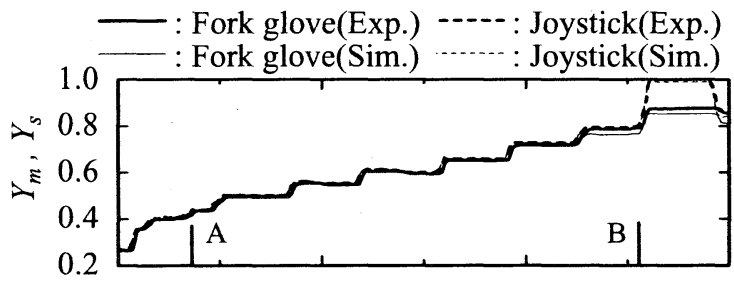

(a) Displacements of joystick and fork glove

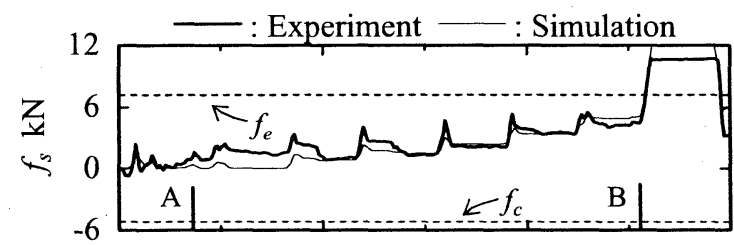

(b) Driving force of fork glove

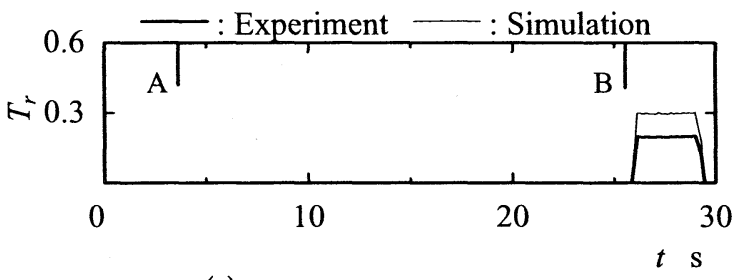

(c) Reaction torque to joystick

Figure 4 Grasping a tire step by step

absent $\left(f_{e}=7.5 \mathrm{kN}, f_{c}=-4.8 \mathrm{kN}\right)$. The maximum driving force to the cylinder in expanding and contracting motions are denoted as $f_{e_{-} \max }$ and $f_{c_{-} \max }\left(f_{e_{-} \max }=11.7 \mathrm{kN}\right.$, $\left.f_{c_{-} \max }=-6.8 \mathrm{kN}\right)$, respectively. Hereafter $f_{e}, f_{c}$ are called threshold driving forces.

It can be inferred from Eqs. (5) and (6) that, when the 
external force is absent in the fork glove, the operation of the joystick becomes smooth because the reaction torque to the master system is zero. When the external force exists, on the other hand, the operator can not necessarily feel the grasping moment well, because the driving force $f_{s}$ does not necessarily exceeds the region of $f_{c} \leq f_{s} \leq f_{e}$.

This unsatisfactory situation occurs in a task when the fork glove grasps a soft object in a slow velocity. As an example for demonstrating such a case, an experimental result obtained in the task of grasping a tire is shown in Fig. 4 (a), (b), (c) along with the corresponding result of simulation. First, displacements of the joystick and the fork glove, $Y_{m}$ and $Y_{s}$, are shown in Fig. 4(a) against time $t$ in the abscissa. The short vertical line marked by $A$ in the figure indicates the starting point of the task. After starting the point $\mathrm{A}$, as shown in the figure, the task of grasping the tire continues in a manner that the grasping force increases step by step and then, at the point B, the tire is crushed completely. From this figure, it is observed that the slave follows the master considerably well. It is also observed that the experimental result agree well with the simulated one. Second, the waveform of the driving force to the fork glove $f_{s}$ is shown in Fig. 4(b). Two broken lines in the figure denote the threshold driving forces $f_{e}, f_{c}$. As seen in the figure, the driving force $f_{s}$ attains a large value after passing the point $\mathrm{B}$ at which the tire is completely crushed. Third, the waveform of the reaction torque to the joystick $T_{r}$ is shown in Fig. 4(c). From the figure, it is observed that the reaction torque $T_{r}$ is not appearing except for the region after the point $B$. In this study, we focus on such a problem as seen here and aim at overcoming the problem.

This problem is considered to be originated from the next reasons. In the control method based on Eq. (6), each value of threshold driving forces $f_{e}, f_{c}$ is treated as constant. For this reason, the reaction torque $T_{r}$ can appear as seen in Fig. 4(c) only when the driving force of the fork glove $f_{s}$ exceeds the threshold $f_{e}$ as seen in Fig. 4(b). On the other hand, in the region of $f_{c} \leq f_{s} \leq f_{e}$, the reaction torque does not appear despite that the fork glove is grasping a task object, since the gain $T$ becomes zero in this situation.

5.2 Improved method for the method of variable-gain symmetric-position

In order to overcome the problem in the previous method, we propose in this section an improved method for the previous one.

In the beginning, the driving force of the hydraulic cylinder $f_{s}$ was measured along with the piston velocity $\dot{y}_{s}$. Thus obtained $\dot{y}_{s}-f_{s}$ characteristics which is denoted by the mark - in Fig.5. As shown in the figure, the driving force $f_{s}$ is clearly indicated as a function of the velocity $\dot{y}_{s}$. Consequently it is better to adopt a variable

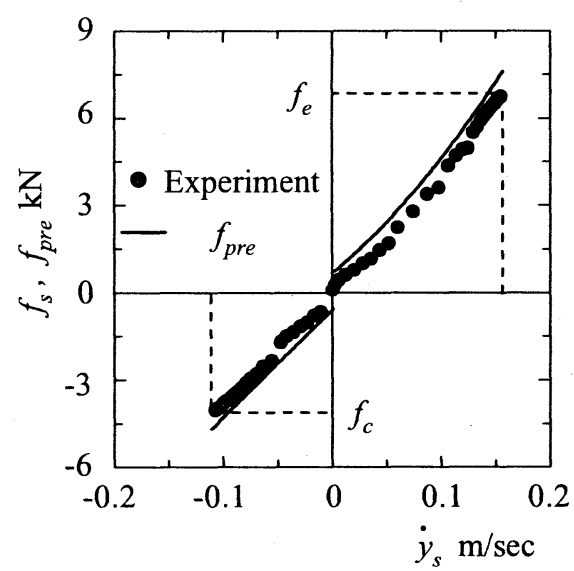

Figure 5 Characteristics of velocity $\dot{y}_{s}$ vs. driving force of $f_{\mathrm{s}}$

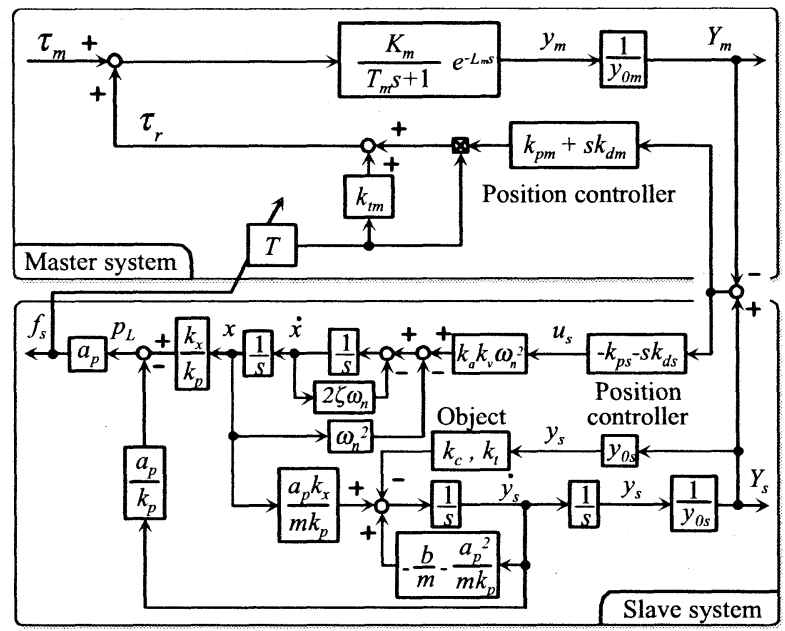

Figure 6 Block diagram of proposed method control

threshold changing with the velocity $\dot{y}_{s}$ for the threshold of the reaction torque to the joystick $\tau_{r}$, instead of fixed thresholds $f_{e}, f_{c}$ as treated in the previous method. In the improved method, therefore, we adopt the variable threshold $f_{\text {pre }}$ as shown by the thin line in Fig.5. In setting the thin line characteristics, as shown in the figure, slightly upward and downward offsets from the measured characteristics are introduced by taking an influence of changing in oil temperature into consideration. As a result, the threshold $f_{\text {pre }}$ is described by Eq. (7).

$$
\begin{cases}f_{\text {pre }}=\left(89 \dot{y}_{s}^{2}+307 \dot{y}_{s}+6\right) \times 100 & \left(\dot{y}_{s} \geq 0\right) \\ f_{\text {pre }}=\left(-59 \dot{y}_{s}^{2}+363 \dot{y}_{s}-6\right) \times 100 & \left(\dot{y}_{s}<0\right)\end{cases}
$$


By applying Eq. (7), it is expected that the new method is able to deal with a grasping motion of time dependence, e.g. slow-moving grasping, and also to feed back sensitively a reaction force of an external force.

In the new method, another improvement as stated in the next is introduced. In the previous method, the reaction torque to the joysticks $\tau_{r}$ is, as shown in Eq. (5), described by the relation depending on the displacement difference between the master and the slave. In this situation, as in a case of grasping a tire, we encounter a problem that the reaction torque to the joystick becomes excessively small when the difference becomes very small. In order to solve this problem, we add the new term $\left(T k_{t m}\right)$ of velocity-independent to the right hand side of Eq. (5). As a consequence, the reaction torque algorithm of the improved method is written by Eq. (8).

$$
\begin{aligned}
& \tau_{r}= T k_{p m}\left(Y_{s}-Y_{m}\right)+T k_{d m}\left(\dot{Y}_{s}-\dot{Y}_{m}\right)+T k_{t m} \\
& T= \begin{cases}0 \quad & \left(\left|f_{s}\right| \leq\left|f_{p r e}\right|\right) \\
0<\frac{f_{s}-f_{p r e}}{f_{e_{-} \max }-f_{p r e}} \leq 1 & \left(f_{s}>0 \cap f_{s}>f_{p r e}\right) \\
0<\frac{f_{s}-f_{p r e}}{f_{c_{-} \max }-f_{p r e}} \leq 1 & \left(f_{s}<0 \cap f_{s}<f_{p r e}\right)\end{cases}
\end{aligned}
$$

As shown in Eq. (9), the gain $T$ changes its value with the change in the threshold $f_{\text {pre }}$.

The features of the improved method based on Eqs.(8),(9) are stated as follows, for example, in the case when the piston tends to expanding direction. If the driving force to the fork glove $f_{s}$ is bigger than the threshold $f_{\text {pre }}$ given by Eq. (9), the difference between them is regarded as the external force to the piston, and thus the corresponding reaction torque can be generated. It is therefore expected in this method that the operator can feel the grasping moment very well, even if he is doing a slow-moving grasping of soft object.

In Fig. 6, the block diagram of the master slave system equipped with the new method is shown.

\section{Experiments and Simulation}

In this chapter, applying the new method to the master slave system, we confirm the availability of the method by experiment and simulation.

Corresponding to the results shown in Fig. 4, a task of grasping a tire step by step with slow velocity was tested by the new method and their results are shown in Fig. 7 . In the figure, the waveform of the gain $T$ is included in Fig. 7(d). As shown in the figure, a subtle change in gain $T$ is appeared as a result of adopting the variable threshold $f_{\text {pre }}$ with velocity $\dot{y}_{s}$. As shown in Fig.7(c), the reaction torque $T_{r}$ is prominence in comparison with

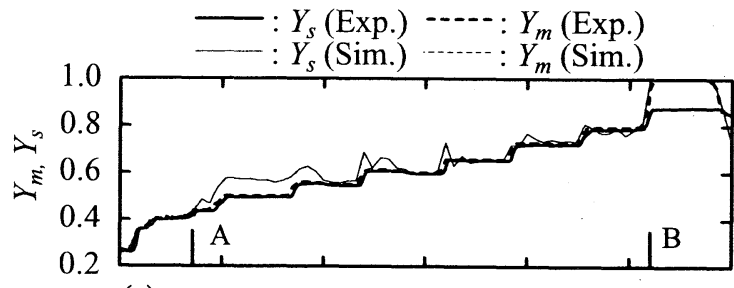

(a) Displacements of joystick and fork glove

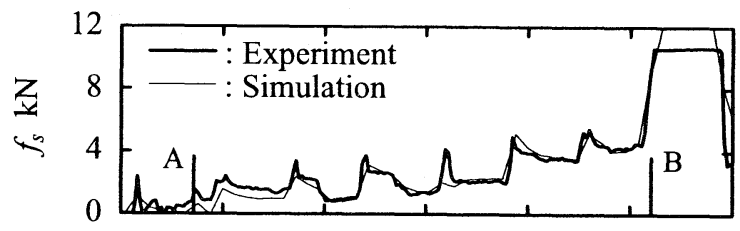

(b) Driving force of fork glove

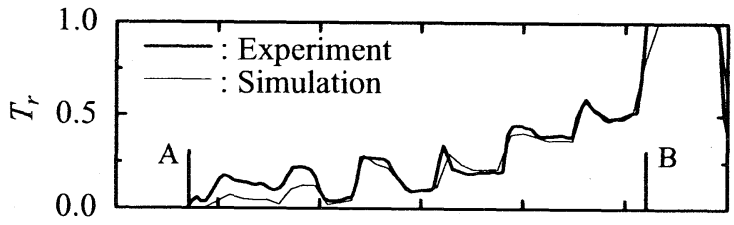

(c) Reaction torque to joystick

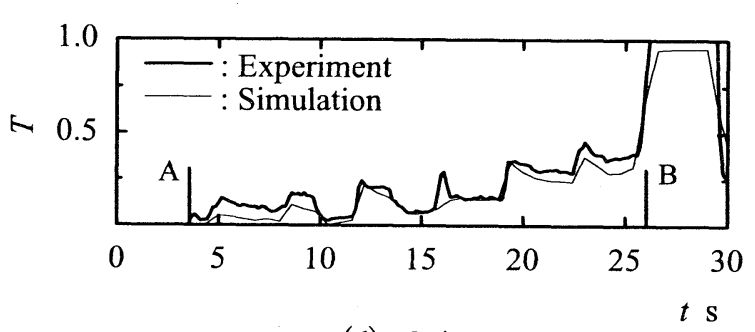

(d) Gain $T$

Figure 7 Grasping a tire step by step

Fig.4(c), because the effect of gain $T$ is satisfactorily reflected on the reaction torque to the joystick. From Fig. 7 , therefore, it can be said that the usefulness of the new method was confirmed. Next, in order to complement this result, another result for grasping a hard object is shown below.

As an example for demonstrating such a case, a task of grasping a concrete block twice is introduced and the result is shown in Figs.8(a) to (c). In these figures, the task consists of two parts: Task 1 and Task 2. In Task 1, the operator grasps the block tightly between $A_{1}$ and $B_{1}$. In Task 2, the operator grasps it softly between $A_{2}$ and $B_{2}$. As shown in Fig.8(a), in Task 1, a large displacement difference between the master and the slave appears, whereas in Task 2, a small displacement difference appears. As shown in Fig.8(b), a large driving force of the fork glove can be detected both in Task 1 and Task 2 . These behaviors shown in Figs.8(a) and (b) are similar to those obtained in the previous method. However it can 


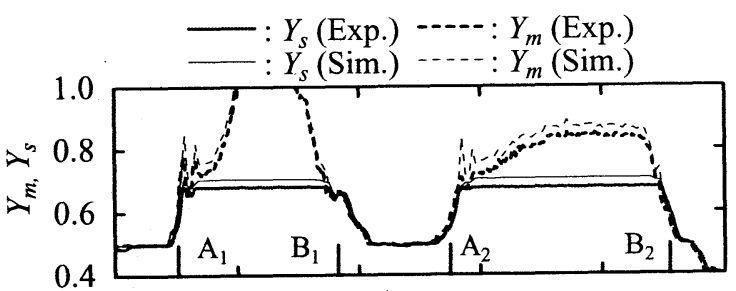

(a) Displacements of joystick and fork glove

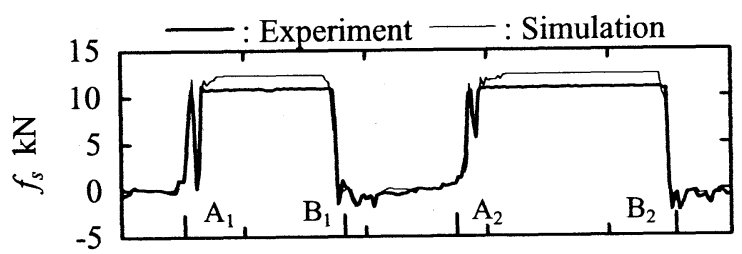

(b) Driving force of fork glove

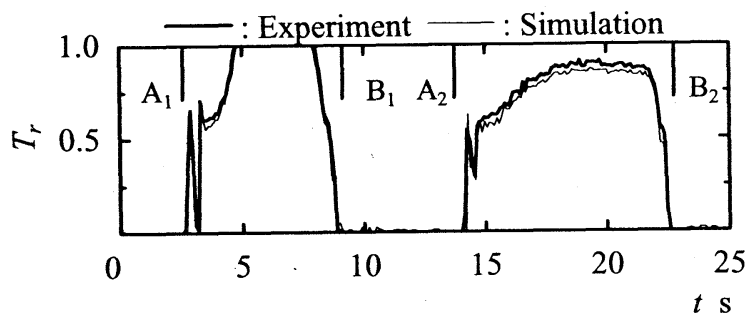

(c) Reaction torque to joystick

Figure 8 Grasping a concrete block

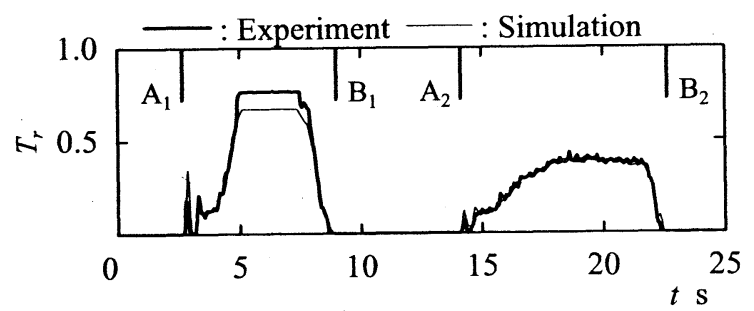

Figure 9 Reaction torque to joystick (Previous method)

be said that the effect of applying the improved method is appearing in the reaction torque to the joystick $T_{r}$ as shown in Fig.8(c). The corresponding reaction torque $T_{r}$ obtained in the previous method is shown in Fig. 9. By comparing Fig. 8(c) and Fig. 9, we can say as follows: first, a relatively large reaction torque is generated to the operator in the improved method in comparison with in the previous one, and second, as shown in Fig. 8(c), the waveform of $T_{r}$ has a sharp increase at the points of $A_{1}$ and $A_{2}$ corresponding to the instances when the operator grasps the block (the sensitivity of force is important at these instances). As a reflection of this fact, in the improved method, the operator could feel the grasping moment very well.

\section{Conclusion}

In a tele-robotic hydraulic construction robot system, the operator needs to feel a realistic sense of task force which is brought about from a feedback force of the fork glove. For this purpose, in the previous study, we proposed a control method named as the variable-gain symmetric-position control. However, a weak point was found in this method. Namely, the operator was not able to feel a realistic sense of the task force when he was grasping a soft object in comparatively a slow velocity. In order to overcome this problem, in this study, we proposed an improved method to the previous one so that the operator can feel enough reaction force. The features of this method are as follows;

(1) In the new method, we adopted a variable threshold changing with the velocity $\dot{y}_{s}$ for the threshold of the reaction torque to the joystick $\tau_{r}$, instead of fixed thresholds $f_{e}, f_{c}$ as treated in the previous method. As a consequence, it is expected that the new method is able to deal with a grasping motion of time dependence, e.g. slow-moving grasping, and also to feed back sensitively a reaction force of an external force.

(2) In the previous method, we encountered a problem that the reaction torque to the joystick becomes excessively small when the difference becomes very small, as in a case of grasping a tire. In order to solve this problem, in the new method, we added a new term $\left(T k_{t m}\right)$ of velocity-independent in the reaction torque algorithm.

We applied the new method to the master slave system and then practiced two tasks of, first, grasping a tire step by step with slow velocity, second, grasping a concrete block tightly and softly. As a result of experiment and simulation for these tasks, the availability of the improved method was confirmed.

\section{References}

1. Y. Yokokohji, Control Theory of Master-slave System, JRSJ, 1993, 11-6, pp.26-34

2. H. Yamada, S. Mukota, Dingxuan, Z. and T. Muto, Construction Telerobot System with Virtual Reality ( Development of a bilateral construction robot), Proc.of VSMM98, 1998, Vol.1, pp.152-157

3. T. Muto, Dynamics and Control of Actuators, Corona Pub., 1992, pp.101-140

4. M. Minamoto, K. Matsunaga, M. Shinohara, H. Kawasaki, K. Yoshinaga: Development of Remote Controlled Robot for Construction Machine, Transactions of VRSJ, Vol.7, No.1, pp.9-14, 2002.

5. H. Hoshino, K. Suzuki, T. Nishiyama, K. Sawada: Development of Tele-existence Visual Display for Humanoid Robot Control, Transactions of VRSJ, Vol.7, No.1, pp.39-48, 2002. 